MIT-CTP-3843

\title{
A Yangian Double for the AdS/CFT Classical r-matrix
}

\author{
SAnefumi Moriyama ${ }^{1,2 *}$ and Alessandro Torrielli ${ }^{1 \dagger}$ \\ ${ }^{1}$ Center for Theoretical Physics, Massachusetts Institute of Technology, \\ Cambridge, MA02139, USA \\ ${ }^{2}$ Graduate School of Mathematics, Nagoya University, \\ Nagoya 464-8602, Japan
}

June, 2007

\begin{abstract}
We express the classical r-matrix of AdS/CFT in terms of tensor products involving an infinite family of generators, which takes a form suggestive of the universal expression obtained from a Yangian double. This should provide an insight into the structure of the infinite dimensional symmetry algebra underlying the integrability of the model, and give a clue to the construction of its universal $\mathrm{R}$-matrix. We derive the commutation relations under which the algebra of these new generators close.
\end{abstract}

*moriyama@math.nagoya-u.ac.jp

†torriell@mit.edu 


\section{Introduction}

Motivated by the AdS/CFT correspondence, integrable structures were discovered on both the gauge theory side [1] and the string theory side [2] (see [3] for reviews). Among the main results has come the derivation of a scattering matrix [4, 5] whose tensorial structure turns out to be completely fixed by the centrally extended $\mathfrak{s u}(2 \mid 2) \oplus \mathfrak{s u}(2 \mid 2)$ symmetry of the problem in its fundamental (2|2) matrix representation. The additional scalar (dressing) factor [6] is constrained by the Hopf-algebraic analog of crossing symmetry [7]. Remarkable advances in the exact determination of this phase factor have been recently made [8, 9], as well as progress in understanding the nature of the infinite dimensional symmetry algebra underlying the planar integrability of the model [10, 11, 12]. In [13], in particular, a Yangian symmetry has been advocated, and the hope for the existence of a universal R-matrix which could reproduce the AdS/CFT scattering matrix upon choosing an appropriate representation, has been put on somewhat firmer grounds. The importance of having a universal form for the R-matrix, namely, an expression purely in terms of generators of the corresponding symmetry algebra independent of the specific representation chosen, comes from two basic facts. The first one, is a better insight into the underlying symmetry responsible for the integrability of the model.

In particular, factorizing the R-matrix explicitly into an infinite sum of tensor products of abstract generators allows to directly read off the symmetry algebra. The operators appearing respectively on the left and on the right hand side of the tensor product are dual to each other, and form a double in Drinfeld's sense. The second useful fact, is the accessibility to all possible scattering matrices for any representations with a single expression. In other words, when taken in the appropriate representation, the universal R-matrix can reduce to the one for the scattering of a bound state from an elementary excitation, or between two bound states. The representations for bound states have been studied in [14].

The presence of the Hopf algebra structure found in [15] precisely leads to the expectation of the existence of an infinite dimensional bialgebra equipped with a triangular structure by the above R-matrix. In order to gain a better insight into this construction, it is useful to study the classical limit of the R-matrix, namely a deformation around the identity: the so-called classical r-matrix, which is a solution of the classical Yang-Baxter equation. The relevance of the classical r-matrix relies on the fact that traditionally one can reconstruct the full quantum R-matrix from the information encoded in its classical limit. This is done by means of powerful theorems [16], which also allow a classification of the possible symmetry structures arising. Roughly speaking, this procedure should correspond to some analog of the exponential map between Lie algebras and Lie groups. For a detailed explanation and motivations of the importance of the classical Yang-Baxter equation, the reader is referred to [17].

The program of analyzing the algebraic structure of the classical r-matrix was initiated in [18], where also the residue at its simple pole at the origin was computed. This revealed the 
appearance of the Casimir of the $\mathfrak{g l}(2 \mid 2)$ superalgebra. Even though a rigorous application of the above mentioned theorems is elusive, the properties of the residue suggest that some version of the standard reconstruction theorems might still work. The plan of this paper is precisely to extract from the classical r-matrix as much information as possible about its universal form. The idea is to obtain a rewriting, which allows us to read the form of the symmetry generators directly from the r-matrix.

In order to do that, we will look for what is called Drinfeld's second realization of Yangians [19]. That is, instead of a realization of the type discussed in [13], given in terms of Lie algebra generators and additional generators constructed recursively (Drinfeld's first realization), we will look for a set of generators parametrized by an integer label, which simultaneously realize the whole Yangian algebra. These generators are traditionally the ones which are employed for constructing the universal R-matrix. For further details about such construction we refer to [20], and to [21] for recent progress along these lines. The goal of the present paper is to read these generators from a rewriting of the classical r-matrix in a form suggestive of a Yangian double, and compute the commutation relations they satisfy, which should become the defining relations of the desired Yangian algebra. We remind that only on the double of the Yangian one can have a well-defined quasi-triangular structure [20].

Let us explain the method we will use in a simple example, namely Yang's classical rmatrix. This is a solution of the classical Yang-Baxter equation of the form

$$
r=\frac{C}{x_{2}-x_{1}}
$$

where $C$ is the Casimir of $\mathfrak{g}$, with $\mathfrak{g}$ being a Lie algebra 1 . One can extract information about the generators of the infinite dimensional symmetry algebra by factorizing it in a geometric sum, $1 /\left(x_{2}-x_{1}\right)=\sum_{n=0}^{\infty} x_{1}^{n} x_{2}^{-n-1}$. If we now express the Casimir in terms of an orthonormal basis $C=T^{a} \otimes T^{a}$, we can see that $r$ takes the form

$$
r=\sum_{n=0}^{\infty} T_{n}^{a} \otimes T_{-n-1}^{a},
$$

where the generators $T_{n}^{a}=T^{a} x^{n}$ are taken in the evaluation representation on both factors of the tensor product. We refer to [17] for a description of the mathematical consequences of such construction in the theory of Lie bialgebras.

We would like to follow the same strategy for the present case. The main difference is that we will have sometimes to expand two denominators, according to the two poles discussed in

\footnotetext{
${ }^{1}$ It is very simple to realize that (1.1) is a solution of $\left[r_{12}, r_{13}\right]+\left[r_{12}, r_{23}\right]+\left[r_{13}, r_{23}\right]=0$, when one remembers that the Casimir operator commutes with the trivial coproduct of the Lie algebra generators, and $r$ lives in $\mathfrak{g} \otimes \mathfrak{g}$.
} 
[18], one at $1 /\left(x_{2}-x_{1}\right)$, one at $1 /\left(x_{1} x_{2}-1\right)$. Nevertheless, we will find that the complicatedlooking expressions can be put into a rather simple and suggestive form:

$$
\begin{aligned}
& r=\sum_{n=0}^{\infty}\left(\mathfrak{Q}_{a, n}^{\alpha} \otimes \widehat{\mathfrak{S}}^{a}{ }_{\alpha,-n-1}-\mathfrak{S}_{\alpha, n}^{a} \otimes \widehat{\mathfrak{Q}}^{\alpha}{ }_{a,-n-1}+\mathfrak{C}_{n} \otimes \widehat{\mathfrak{I}}_{-n-1}+\mathfrak{I}_{n} \otimes \widehat{\mathfrak{C}}_{-n-1}\right. \\
& \left.+\left(\mathfrak{R}_{b, n}^{a} \otimes \widehat{\mathfrak{R}}^{b}{ }_{a,-n-1}-\mathfrak{R}_{b,-n-1}^{a} \otimes \widehat{\mathfrak{R}}_{a, n}^{b}\right)-\left(\mathfrak{L}_{\beta, n}^{\alpha} \otimes \widehat{\mathfrak{L}}^{\beta}{ }_{\alpha,-n-1}-\mathfrak{L}^{\alpha}{ }_{\beta,-n-1} \otimes \widehat{\mathfrak{L}}^{\beta}{ }_{\alpha, n}\right)\right)
\end{aligned}
$$

where the generators will be defined in the main text. This form is reminiscent of what one expects from the structure of a Yangian double. We will derive these infinite families of generators in a particular representation, which directly emerges from the classical r-matrix. We call the subscript $n$ the level of the Yangian. At level-zero we recover the original Lie superalgebra generators.

The generator $\mathfrak{I}$ appearing in (1.3) is proportional to $\operatorname{diag}(1,1,-1,-1)$, and extends the Cartan subalgebra of $\mathfrak{s l}(2 \mid 2)$ to $\mathfrak{g l}(2 \mid 2)$. Such an extension needs to be introduced on general grounds. Whenever, in fact, the Cartan matrix of a Lie superalgebra is degenerate (as in the present case for $\mathfrak{s u}(2 \mid 2)$ ), one needs to introduce an additional Cartan generator in order to make such matrix non-degenerate, and be able to invert it. The inverse of the Cartan matrix extended in this way will appear in the universal R-matrix, together with the additional Cartan generator [20]2. In the present case, we follow the discussion in [23]: in that paper, the original $\mathfrak{s l}(2 \mid 2)$ Cartan subalgebra consists of the generators $H_{1}=\operatorname{diag}(-1,0,-1,0)$, $H_{2}=\operatorname{diag}(0,1,1,0)$ and $H_{3}=\operatorname{diag}(0,-1,0,-1)$. One has to introduce an additional $H_{4}=$ $\operatorname{diag}(-1,0,0,1)$ which completes the algebra to $\mathfrak{g l}(2 \mid 2)$. Then the extended Cartan matrix reads:

$$
a=\left(\begin{array}{cccc}
0 & 1 & 0 & 1 \\
1 & 0 & -1 & 0 \\
0 & -1 & 0 & 1 \\
1 & 0 & 1 & 0
\end{array}\right)
$$

If we re-express these generators in more familiar notation in terms of $\mathfrak{R}=\operatorname{diag}(1,-1,0,0)$, $\mathfrak{L}=\operatorname{diag}(0,0,1,-1), \mathfrak{C}=1 / 2 \operatorname{diag}(1,1,1,1)$ and $\mathfrak{I}=1 / 2 \operatorname{diag}(1,1,-1,-1)$, the quadratic form reduces to

$$
\left(a^{-1}\right)^{i j} H_{i} H_{j}=\frac{1}{2}\left(\mathfrak{R}^{2}-\mathfrak{L}^{2}\right)+2 \mathfrak{C} \mathfrak{I}
$$

which is reminiscent of the form (1.3). We will find that the coefficient of $\mathfrak{I}_{n}$ vanishes for $n=0$. This is consistent with the result of [18]. There, an analysis of the poles of the classical r-matrix was performed, and the appearance of the Casimir of the superalgebra $\mathfrak{g l}(2 \mid 2)$ in the residue at $x_{1}=x_{2}$ was shown. One thing to notice is that, nevertheless, the terms of

\footnotetext{
${ }^{2}$ An alternative extension by $\mathfrak{s l}(2)$ automorphisms has been considered in [5, 22, 13. In particular in [22] the Casimir operator for such an extension was considered.
} 
the R-matrix responsible for the exchange between two bosons and two fermions, namely $C_{12}$ and $F_{12}$ in [4], do not contribute to the residue at this pole. Therefore, the residue has an additional symmetry, corresponding to the trivial coproduct of the generator $\operatorname{diag}(1,1,-1-1)$, which enhances the algebra to $\mathfrak{g l}(2 \mid 2)$. However, this symmetry is neither of the full R-matrix, nor of the classical r-matrix, precisely due to these terms.

The plan of the paper is as follows: In section 2 we review the properties of the classical r-matrix, introducing the conventions needed. In section 3 we perform our rewriting of the entries of the classical r-matrix in terms of generators of a Yangian double, starting from the easier non-diagonal part, and ending with the diagonal one. The new generators are also introduced, whose commutation relations are presented in section 4 . We conclude with comments on the main directions of future development.

\section{Review of the classical r-matrix}

Our starting point for the rewriting (1.3) is the classical r-matrix given in [18]. Though the $\mathfrak{s u}(\mathbf{1} \mid 2)$ basis was adopted there, it turned out to be the so-called string basis [11] that makes direct contact with the string theory computation. Here we would like to briefly review the classical r-matrix in the string basis. The R-matrix $\mathcal{R}=\Pi \circ \mathcal{S}$ is constructed from the graded permutation $\Pi$ and the S-matrix $\mathcal{S}$ given in [4, 5] (see also the comments in [13]):

$$
\begin{aligned}
\mathcal{R}_{12}\left|\phi_{1}^{a} \phi_{2}^{b}\right\rangle & =\frac{1}{2}\left(A_{12}-B_{12}\right) \frac{U_{1}}{U_{2}}\left|\phi_{1}^{a} \phi_{2}^{b}\right\rangle+\frac{1}{2}\left(A_{12}+B_{12}\right) \frac{U_{1}}{U_{2}}\left|\phi_{1}^{b} \phi_{2}^{a}\right\rangle+\frac{1}{2} C_{12} U_{1} \epsilon^{a b} \epsilon_{\alpha \beta}\left|\psi_{1}^{\alpha} \psi_{2}^{\beta}\right\rangle \\
\mathcal{R}_{12}\left|\psi_{1}^{\alpha} \psi_{2}^{\beta}\right\rangle & =-\frac{1}{2}\left(D_{12}-E_{12}\right)\left|\psi_{1}^{\alpha} \psi_{2}^{\beta}\right\rangle-\frac{1}{2}\left(D_{12}+E_{12}\right)\left|\psi_{1}^{\beta} \psi_{2}^{\alpha}\right\rangle-\frac{1}{2} F_{12} \frac{1}{U_{2}} \epsilon^{\alpha \beta} \epsilon_{a b}\left|\phi_{1}^{a} \phi_{2}^{b}\right\rangle \\
\mathcal{R}_{12}\left|\phi_{1}^{a} \psi_{2}^{\beta}\right\rangle & =G_{12} \frac{1}{U_{2}}\left|\phi_{1}^{a} \psi_{2}^{\beta}\right\rangle+H_{12}\left|\psi_{1}^{\beta} \phi_{2}^{a}\right\rangle \\
\mathcal{R}_{12}\left|\psi_{1}^{\alpha} \phi_{2}^{b}\right\rangle & =L_{12} U_{1}\left|\psi_{1}^{\alpha} \phi_{2}^{b}\right\rangle+K_{12} \frac{U_{1}}{U_{2}}\left|\phi_{1}^{b} \psi_{2}^{\alpha}\right\rangle
\end{aligned}
$$


with $U=\sqrt{x^{+} / x^{-}}$and $A_{12}, B_{12}, \ldots$ given by

$$
\begin{gathered}
A_{12}=\frac{x_{2}^{+}-x_{1}^{-}}{x_{2}^{-}-x_{1}^{+}}, \quad B_{12}=\frac{x_{2}^{+}-x_{1}^{-}}{x_{2}^{-}-x_{1}^{+}}\left(1-2 \frac{1-g^{2} / 2 x_{1}^{+} x_{2}^{-}}{1-g^{2} / 2 x_{1}^{-} x_{2}^{-}} \frac{x_{2}^{+}-x_{1}^{+}}{x_{2}^{+}-x_{1}^{-}}\right), \\
C_{12}=\frac{g^{2} \gamma_{1} \gamma_{2}}{\alpha x_{1}^{+} x_{2}^{+}} \frac{1}{1-g^{2} / 2 x_{1}^{-} x_{2}^{-}} \frac{x_{2}^{+}-x_{1}^{+}}{x_{2}^{-}-x_{1}^{+}}, \\
D_{12}=-1, \quad E_{12}=-\left(1-2 \frac{1-g^{2} / 2 x_{1}^{-} x_{2}^{+}}{1-g^{2} / 2 x_{1}^{+} x_{2}^{+}} \frac{x_{2}^{-}-x_{1}^{-}}{x_{2}^{-}-x_{1}^{+}}\right) \\
F_{12}=-\frac{2 \alpha\left(x_{1}^{+}-x_{1}^{-}\right)\left(x_{2}^{+}-x_{2}^{-}\right)}{1-g^{2} / 2 x_{1}^{+} x_{2}^{+}} \frac{x_{2}^{-}-x_{1}^{-}}{x_{2}^{-}-x_{1}^{+}} \\
G_{12}=\frac{x_{2}^{+}-x_{1}^{+}}{x_{2}^{-}-x_{1}^{+}}, \quad x_{12}^{-} x_{2}^{-}=\frac{\gamma_{1}}{\gamma_{2}} \frac{x_{2}^{+}-x_{2}^{-}}{x_{2}^{-}-x_{1}^{+}} \\
L_{12}=\frac{x_{2}^{-}-x_{1}^{-}}{x_{2}^{-}-x_{1}^{+}}, \quad K_{12}=\frac{\gamma_{2}}{\gamma_{1}} \frac{x_{1}^{+}-x_{1}^{-}}{x_{2}^{-}-x_{1}^{+}}
\end{gathered}
$$

We adopt the parametrization of [8] for the variables $x^{ \pm}$:

$$
x^{ \pm}(x)=\frac{x}{2 \zeta}\left(\sqrt{1-\frac{\zeta^{2}}{\left(x-x^{-1}\right)^{2}}} \pm i \frac{\zeta}{x-x^{-1}}\right),
$$

and we take $\zeta=1 /(\sqrt{2} g)\left(=2 \pi / \sqrt{\lambda}\right.$ in terms of the 't Hooft coupling constant $\left.\lambda=g_{\mathrm{YM}}^{2} N\right)$ as a deformation parameter, namely expand all formulas around $\zeta=0$ keeping $x$ fixed. This corresponds to the near BMN limit [24]. The classical r-matrix is defined by the infinitesimal deviation from unity of the R-matrix:

$$
\mathcal{R}_{12}=1+i \zeta r_{12} .
$$

After some computations we find that it is given by

$$
\begin{aligned}
r_{12}\left|\phi_{1}^{a} \phi_{2}^{b}\right\rangle= & \frac{\left(x_{1}^{2}+x_{2}^{2}\right)\left(x_{1}^{2} x_{2}^{2}+1\right)-4 x_{1}^{2} x_{2}^{2}}{\left(x_{2}-x_{1}\right)\left(x_{1} x_{2}-1\right)\left(x_{1}^{2}-1\right)\left(x_{2}^{2}-1\right)}\left|\phi_{1}^{a} \phi_{2}^{b}\right\rangle+\frac{2 x_{1} x_{2}}{\left(x_{2}-x_{1}\right)\left(x_{1} x_{2}-1\right)}\left|\phi_{1}^{b} \phi_{2}^{a}\right\rangle \\
& +\frac{\gamma_{1} \gamma_{2}}{i \zeta \alpha} \frac{1}{x_{1} x_{2}-1} \epsilon^{a b} \epsilon_{\alpha \beta}\left|\psi_{1}^{\alpha} \psi_{2}^{\beta}\right\rangle, \\
r_{12}\left|\psi_{1}^{\alpha} \psi_{2}^{\beta}\right\rangle= & \frac{2 x_{1} x_{2}}{\left(x_{2}-x_{1}\right)\left(x_{1} x_{2}-1\right)}\left|\psi_{1}^{\alpha} \psi_{2}^{\beta}\right\rangle-\frac{2 x_{1} x_{2}}{\left(x_{2}-x_{1}\right)\left(x_{1} x_{2}-1\right)}\left|\psi_{1}^{\beta} \psi_{2}^{\alpha}\right\rangle \\
& -\frac{4 \zeta \alpha}{i \gamma_{1} \gamma_{2}} \frac{x_{1}^{2} x_{2}^{2}}{\left(x_{1}^{2}-1\right)\left(x_{2}^{2}-1\right)\left(x_{1} x_{2}-1\right)} \epsilon^{\alpha \beta} \epsilon_{a b}\left|\phi_{1}^{a} \phi_{2}^{b}\right\rangle, \\
r_{12}\left|\phi_{1}^{a} \psi_{2}^{\beta}\right\rangle= & \frac{x_{2}\left(x_{2}+x_{1}\right)}{\left(x_{2}-x_{1}\right)\left(x_{2}^{2}-1\right)}\left|\phi_{1}^{a} \psi_{2}^{\beta}\right\rangle+\frac{\gamma_{1}}{\gamma_{2}} \frac{2 x_{2}^{2}}{\left(x_{2}-x_{1}\right)\left(x_{2}^{2}-1\right)}\left|\psi_{1}^{\beta} \phi_{2}^{a}\right\rangle, \\
r_{12}\left|\psi_{1}^{\alpha} \phi_{2}^{b}\right\rangle= & \frac{x_{1}\left(x_{2}+x_{1}\right)}{\left(x_{2}-x_{1}\right)\left(x_{1}^{2}-1\right)}\left|\psi_{1}^{\alpha} \phi_{2}^{b}\right\rangle+\frac{\gamma_{2}}{\gamma_{1}} \frac{2 x_{1}^{2}}{\left(x_{2}-x_{1}\right)\left(x_{1}^{2}-1\right)}\left|\phi_{1}^{b} \psi_{2}^{\alpha}\right\rangle .
\end{aligned}
$$


We would like to rewrite this classical r-matrix in terms of the $\mathfrak{s u ( 2 | 2 )}$ generators $\mathfrak{R}^{a}{ }_{b}$, $\mathfrak{L}^{\alpha}{ }_{\beta}, \mathfrak{Q}^{\alpha}{ }_{a}, \mathfrak{S}^{a}{ }_{\alpha}$ with the central element $\mathfrak{C}$, and their infinite Yangian partners labeled by an integer $n$, whose fundamental representation for $n=0$ is given by

$$
\begin{aligned}
& \mathfrak{R}_{b}^{a}\left|\phi^{c}\right\rangle=\delta_{b}^{c}\left|\phi^{a}\right\rangle-\frac{1}{2} \delta_{b}^{a}\left|\phi^{c}\right\rangle, \\
& \mathfrak{L}_{\beta}^{\alpha}\left|\psi^{\gamma}\right\rangle=\delta_{\beta}^{\gamma}\left|\psi^{\alpha}\right\rangle-\frac{1}{2} \delta_{\beta}^{\alpha}\left|\psi^{\gamma}\right\rangle,
\end{aligned}
$$

and

$$
\begin{aligned}
& \mathfrak{Q}^{\alpha}{ }_{a}\left|\phi^{b}\right\rangle=a \delta_{a}^{b}\left|\psi^{\alpha}\right\rangle, \quad \mathfrak{Q}^{\alpha}{ }_{a}\left|\psi^{\beta}\right\rangle=b \epsilon^{\alpha \beta} \epsilon_{a b}\left|\phi^{b}\right\rangle, \\
& \mathfrak{S}^{a}{ }_{\alpha}\left|\phi^{b}\right\rangle=c \epsilon^{a b} \epsilon_{\alpha \beta}\left|\psi^{\beta}\right\rangle, \quad \mathfrak{S}^{a}{ }_{\alpha}\left|\psi^{\beta}\right\rangle=d \delta_{\alpha}^{\beta}\left|\phi^{a}\right\rangle,
\end{aligned}
$$

as well as

$$
\mathfrak{C}\left|\phi^{a}\right\rangle=C\left|\phi^{a}\right\rangle, \quad \mathfrak{C}\left|\psi^{\alpha}\right\rangle=C\left|\psi^{\alpha}\right\rangle
$$

with $a, b, c, d$ defined by the limit $\zeta \rightarrow 0$ of the corresponding variables introduces in [4] (see also [18])

$$
a=\gamma, \quad b=\frac{2 \zeta \alpha}{i \gamma} \frac{x}{x^{2}-1}, \quad c=\frac{i \gamma}{2 \zeta \alpha} \frac{1}{x}, \quad d=\frac{1}{\gamma} \frac{x^{2}}{x^{2}-1} .
$$

It is understood that $\alpha$ scales as $\zeta^{-1}$ and $\gamma$ is of order 1 in the classical limit. An additional operator $\mathfrak{I}$ whose eigenvalue vanishes for $n=0$ will be introduced later.

\section{Classical r-matrix as a Yangian double}

After reviewing the expression for the classical r-matrix in the previous section, here we would like to embark on our project of rewriting the classical r-matrix (2.5)-(2.8) in terms of generators as in (1.3). Since each sector is independent, we shall start with the easier off-diagonal sector, and then turn to the diagonal sector.

\subsection{Fermionic sector}

First, let us concentrate on the combination of two fermionic generators, which only affect the last terms in (2.5)-(2.8). For this purpose we note that half of the coefficients from each term 
can be expressed as

$$
\begin{aligned}
\frac{\gamma_{1} \gamma_{2}}{i 2 \zeta \alpha} \frac{1}{x_{1} x_{2}-1} & =\sum_{n=0}^{\infty} a_{1} x_{1}^{n} \cdot c_{2} x_{2}^{n+1}=-\sum_{n=0}^{\infty} c_{1} x_{1}^{-n} \cdot a_{2} x_{2}^{-n-1}, \\
-\frac{2 \zeta \alpha}{i \gamma_{1} \gamma_{2}} \frac{x_{1}^{2} x_{2}^{2}}{\left(x_{1}^{2}-1\right)\left(x_{2}^{2}-1\right)\left(x_{1} x_{2}-1\right)} & =-\sum_{n=0}^{\infty} b_{1} x_{1}^{-n} \cdot d_{2} x_{2}^{-n-1}=\sum_{n=0}^{\infty} d_{1} x_{1}^{n} \cdot b_{2} x_{2}^{n+1} \\
\frac{\gamma_{1}}{\gamma_{2}} \frac{x_{2}^{2}}{\left(x_{2}-x_{1}\right)\left(x_{2}^{2}-1\right)} & =\sum_{n=0}^{\infty} a_{1} x_{1}^{n} \cdot d_{2} x_{2}^{-n-1}=-\sum_{n=0}^{\infty} c_{1} x_{1}^{-n} \cdot b_{2} x_{2}^{n+1} \\
\frac{\gamma_{2}}{\gamma_{1}} \frac{x_{1}^{2}}{\left(x_{2}-x_{1}\right)\left(x_{1}^{2}-1\right)} & =-\sum_{n=0}^{\infty} b_{1} x_{1}^{-n} \cdot c_{2} x_{2}^{n+1}=\sum_{n=0}^{\infty} d_{1} x_{1}^{n} \cdot a_{2} x_{2}^{-n-1}
\end{aligned}
$$

in an appropriate domain of convergence. Since the action of two fermionic generators on two excitations goes as

$$
\begin{aligned}
\mathfrak{Q}^{\alpha}{ }_{a} \otimes \mathfrak{S}^{a}{ }_{\alpha}\left|\phi_{1}^{b} \phi_{2}^{c}\right\rangle=a_{1} c_{2} \epsilon^{b c} \epsilon_{\beta \gamma}\left|\psi_{1}^{\beta} \psi_{2}^{\gamma}\right\rangle, & \mathfrak{S}^{a}{ }_{\alpha} \otimes \mathfrak{Q}^{\alpha}{ }_{a}\left|\phi_{1}^{b} \phi_{2}^{c}\right\rangle=c_{1} a_{2} \epsilon^{b c} \epsilon_{\beta \gamma}\left|\psi_{1}^{\beta} \psi_{2}^{\gamma}\right\rangle \\
\mathfrak{Q}^{\alpha}{ }_{a} \otimes \mathfrak{S}^{a}{ }_{\alpha}\left|\psi_{1}{ }_{1} \psi_{2}^{\gamma}\right\rangle=-b_{1} d_{2} \epsilon^{\beta \gamma} \epsilon_{b c}\left|\phi_{1}^{b} \phi_{2}^{c}\right\rangle, & \mathfrak{S}^{a}{ }_{\alpha} \otimes \mathfrak{Q}^{\alpha}{ }_{a}\left|\psi_{1}^{\beta} \psi_{2}^{\gamma}\right\rangle=-d_{1} b_{2} \epsilon^{\beta \gamma} \epsilon_{b c}\left|\phi_{1}^{b} \phi_{2}^{c}\right\rangle \\
\mathfrak{Q}^{\alpha}{ }_{a} \otimes \mathfrak{S}^{a}{ }_{\alpha}\left|\phi_{1}^{b} \psi_{2}^{\gamma}\right\rangle=a_{1} d_{2}\left|\psi_{1}^{\gamma} \phi_{2}^{b}\right\rangle, & \mathfrak{S}^{a}{ }_{\alpha} \otimes \mathfrak{Q}^{\alpha}{ }_{a}\left|\phi_{1}^{b} \psi_{2}^{\gamma}\right\rangle=c_{1} b_{2}\left|\psi_{1}^{\gamma} \phi_{2}^{b}\right\rangle \\
\mathfrak{Q}^{\alpha}{ }_{a} \otimes \mathfrak{S}^{a}{ }_{\alpha}\left|\psi_{1}^{\beta} \phi_{2}^{c}\right\rangle=-b_{1} c_{2}\left|\phi_{1}^{c} \psi_{2}^{\beta}\right\rangle, & \mathfrak{S}^{a}{ }_{\alpha} \otimes \mathfrak{Q}^{\alpha}{ }_{a}\left|\psi_{1}^{\beta} \phi_{2}^{c}\right\rangle=-d_{1} a_{2}\left|\phi_{1}^{c} \psi_{2}^{\beta}\right\rangle
\end{aligned}
$$

we find that in the fermionic sector the classical r-matrix can be expressed as

$$
\left.r\right|_{\mathfrak{Q S}}=\sum_{n=0}^{\infty}\left(\mathfrak{Q}_{a, n}^{\alpha} \otimes \widehat{\mathfrak{S}}^{a}{ }_{\alpha,-n-1}-\mathfrak{S}^{a}{ }_{\alpha, n} \otimes \widehat{\mathfrak{Q}}^{\alpha}{ }_{a,-n-1}\right)
$$

with $\mathfrak{Q}^{\alpha}{ }_{a, n}, \widehat{\mathfrak{Q}}^{\alpha}{ }_{a, n}, \mathfrak{S}^{a}{ }_{\alpha, n}$ and $\widehat{\mathfrak{S}}^{a}{ }_{\alpha, n}$ defined by

$$
\begin{aligned}
& \mathfrak{Q}_{a, n}^{\alpha}=\widehat{\mathfrak{Q}}^{\alpha}{ }_{a, n}=\mathfrak{Q}^{\alpha}{ }_{a}\left(x^{n} \Pi_{\mathrm{b}}+x^{-n} \Pi_{\mathrm{f}}\right), \\
& \mathfrak{S}^{a}{ }_{\alpha, n}=\widehat{\mathfrak{S}}^{a}{ }_{\alpha, n}=\mathfrak{S}^{a}{ }_{\alpha}\left(x^{-n} \Pi_{\mathrm{b}}+x^{n} \Pi_{\mathrm{f}}\right) .
\end{aligned}
$$

The operators $\Pi_{\mathrm{b}}$ and $\Pi_{\mathrm{f}}$ are projectors in the bosonic and fermionic subspaces respectively, namely in matrix notation $\Pi_{\mathrm{b}}=\operatorname{diag}(1,1,0,0)$ and $\Pi_{\mathrm{f}}=\operatorname{diag}(0,0,1,1)$. Note that both operators without hats $\mathfrak{Q}^{\alpha}{ }_{a, n}, \mathfrak{S}^{a}{ }_{\alpha, n}$ and operators with hats $\widehat{\mathfrak{Q}}^{\alpha, n}{ }_{a, n}, \widehat{\mathfrak{S}}^{a}{ }_{\alpha, n}$ have the same expressions, though the operators without hats are only defined for $n \geq 0$ while the operators with hats are only defined for $n<0$. Note also that the expression in (3.3) should be regarded as a formal series. After acting on states, we interpret the summation as an analytical continuation from the result obtained in an appropriate domain of convergence. Equivalently, one could act on the r-matrix with the operator $\left(D_{\rho} \otimes 1\right)[23]$, where the operator $D_{\rho}$ multiplies any generator at level $n$ by the representation-independent parameter $\rho^{|n|}$, perform the series in a domain of $\rho$ where one has convergence, and analytically continue to $\rho=1$ at the end. 


\subsection{Bosonic off-diagonal sector}

Now let us turn to the bosonic off-diagonal sector. We would like to rewrite the second terms in (2.5) and (2.6) into the operator doubles. Here the coefficient can be rewritten as

$$
\frac{2 x_{1} x_{2}}{\left(x_{2}-x_{1}\right)\left(x_{1} x_{2}-1\right)}=\sum_{n=0}^{\infty}\left([n+1]_{x_{1}}[n+2]_{x_{2}}-[n+2]_{x_{1}}[n+1]_{x_{2}}\right)
$$

because of

$$
\begin{gathered}
\frac{x_{1} x_{2}}{\left(x_{2}-x_{1}\right)\left(x_{1} x_{2}-1\right)}=\sum_{n=0}^{\infty}[n+1]_{x_{1}} x_{2}^{n+1}=\sum_{n=0}^{\infty}[n+1]_{x_{1}} x_{2}^{-n-1} \\
=-\sum_{n=0}^{\infty} x_{1}^{n+1}[n+1]_{x_{2}}=-\sum_{n=0}^{\infty} x_{1}^{-n-1}[n+1]_{x_{2}} .
\end{gathered}
$$

Here we have introduced a $q$-number $[n]_{q}$ by

$$
[n]_{q}=\frac{q^{n}-q^{-n}}{q-q^{-1}}
$$

Since the action of the operators $\mathfrak{R}_{b}^{a} \otimes \mathfrak{R}^{b}{ }_{a}$ and $\mathfrak{L}^{\alpha}{ }_{\beta} \otimes \mathfrak{L}^{\beta}{ }_{\alpha}$ takes the form

$$
\mathfrak{R}_{b}^{a} \otimes \mathfrak{R}^{b}{ }_{a}\left|\phi_{1}^{c} \phi_{2}^{d}\right\rangle=\left|\phi_{1}^{d} \phi_{2}^{c}\right\rangle-\frac{1}{2}\left|\phi_{1}^{c} \phi_{2}^{d}\right\rangle, \quad \mathfrak{L}_{\beta}^{\alpha} \otimes \mathfrak{L}^{\beta}{ }_{\alpha}\left|\psi_{1}^{\gamma} \psi_{2}^{\delta}\right\rangle=\left|\psi_{1}^{\delta} \psi_{2}^{\gamma}\right\rangle-\frac{1}{2}\left|\psi_{1}^{\gamma} \psi_{2}^{\delta}\right\rangle
$$

we can rewrite the classical r-matrix in this sector as

$$
\begin{aligned}
\left.r\right|_{\mathfrak{R L}}= & \sum_{n=0}^{\infty}\left(\left(\mathfrak{R}_{b, n}^{a} \otimes \widehat{\mathfrak{R}}_{a,-n-1}^{b}-\mathfrak{R}_{b,-n-1}^{a} \otimes \widehat{\mathfrak{R}}_{a, n}^{b}\right)\right. \\
& \left.-\left(\mathfrak{L}_{\beta, n}^{\alpha} \otimes \widehat{\mathfrak{L}}^{\beta}{ }_{\alpha,-n-1}-\mathfrak{L}_{\beta,-n-1}^{\alpha} \otimes \widehat{\mathfrak{L}}^{\beta}{ }_{\alpha, n}\right)\right),
\end{aligned}
$$

with $\mathfrak{R}_{b, n}^{a}, \widehat{\mathfrak{R}}_{b, n}^{a}, \mathfrak{L}_{\beta, n}^{\alpha}$ and $\widehat{\mathfrak{L}}_{\beta, n}^{\alpha}$ defined by

$$
\begin{array}{ll}
\mathfrak{R}_{b, n}^{a}=[n+1]_{x} \mathfrak{R}_{b}^{a}, & \widehat{\mathfrak{R}}_{b, n}^{a}=-[n-1]_{x} \mathfrak{R}_{b}^{a}, \\
\mathfrak{L}_{\beta, n}^{\alpha}=[n+1]_{x} \mathfrak{L}_{\beta}^{\alpha}, & \widehat{\mathfrak{L}}_{\beta, n}^{\alpha}=-[n-1]_{x} \mathfrak{L}^{\alpha}{ }_{\beta} .
\end{array}
$$

In this case, all of $\mathfrak{R}_{b, n}^{a}, \widehat{\mathfrak{R}}_{b, n}^{a}, \mathfrak{L}_{\beta, n}^{\alpha}$ and $\widehat{\mathfrak{L}}_{\beta, n}^{\alpha}$ are defined for both $n \geq 0$ and $n<0$. Note that there are ambiguities in this rewriting because of various expressions in (3.7). Our current choice is partially motivated by its rather symmetric form, and partially by the closure of the commutation relations, which will be the subject of our next section. 


\subsection{Bosonic diagonal sector}

Finally let us consider the diagonal sector. We assume that the Cartan subalgebra of $\mathfrak{s u}(2) \times$ $\mathfrak{s u}(2)$ (generators $\mathfrak{R}_{b}^{a}$ and $\mathfrak{L}_{\beta}^{\alpha}$ ) is already taken care of in (3.10) by suitably completing the set of indices contracted. This is simply due to $\mathfrak{s u}(2) \times \mathfrak{s u}(2)$ covariance of the string basis. The remaining diagonal part we would like to rewrite into the form of a Yangian double is therefore

$$
\begin{aligned}
r_{12}\left|\phi_{1}^{a} \phi_{2}^{b}\right\rangle & =\left[\frac{\left(x_{1}^{2}+x_{2}^{2}\right)\left(x_{1}^{2} x_{2}^{2}+1\right)-4 x_{1}^{2} x_{2}^{2}}{\left(x_{2}-x_{1}\right)\left(x_{1} x_{2}-1\right)\left(x_{1}^{2}-1\right)\left(x_{2}^{2}-1\right)}+\frac{x_{1} x_{2}}{\left(x_{2}-x_{1}\right)\left(x_{1} x_{2}-1\right)}\right]\left|\phi_{1}^{a} \phi_{2}^{b}\right\rangle+\cdots, \\
r_{12}\left|\psi_{1}^{\alpha} \psi_{2}^{\beta}\right\rangle & =\left[\frac{2 x_{1} x_{2}}{\left(x_{2}-x_{1}\right)\left(x_{1} x_{2}-1\right)}-\frac{x_{1} x_{2}}{\left(x_{2}-x_{1}\right)\left(x_{1} x_{2}-1\right)}\right]\left|\psi_{1}^{\alpha} \psi_{2}^{\beta}\right\rangle+\cdots \\
r_{12}\left|\phi_{1}^{a} \psi_{2}^{\beta}\right\rangle & =\frac{x_{2}\left(x_{2}+x_{1}\right)}{\left(x_{2}-x_{1}\right)\left(x_{2}^{2}-1\right)}\left|\phi_{1}^{a} \psi_{2}^{\beta}\right\rangle+\cdots \\
r_{12}\left|\psi_{1}^{\alpha} \phi_{2}^{b}\right\rangle & =\frac{x_{1}\left(x_{2}+x_{1}\right)}{\left(x_{2}-x_{1}\right)\left(x_{1}^{2}-1\right)}\left|\psi_{1}^{\alpha} \phi_{2}^{b}\right\rangle+\cdots .
\end{aligned}
$$

The extra term in the squared parentheses comes from rearranging the operator action as (3.9). Note that the phase of the S-matrix was undetermined in 44. Hence the diagonal sector has the ambiguity of an overall shift, corresponding to an overall scalar factor at the level of the full quantum R-matrix. In this paper, we will still freely add and subtract such terms when needed, but they should later be determined by some generalized crossing symmetry emerging from the construction.

Inspired by the argument in the introduction, we would like to make use of the following generators

$$
\begin{aligned}
& \mathfrak{C}_{n}\left|\phi^{a}\right\rangle=C_{n}\left|\phi^{a}\right\rangle, \quad \mathfrak{C}_{n}\left|\psi^{\alpha}\right\rangle=C_{n}\left|\psi^{\alpha}\right\rangle, \\
& \mathfrak{I}_{n}\left|\phi^{a}\right\rangle=I_{n}\left|\phi^{a}\right\rangle, \quad \mathfrak{I}_{n}\left|\psi^{\alpha}\right\rangle=-I_{n}\left|\psi^{\alpha}\right\rangle .
\end{aligned}
$$

If we assume the classical r-matrix can be expressed as

$$
\left.r\right|_{\mathfrak{C I}}=\sum_{n=0}^{\infty}\left(\mathfrak{C}_{n} \otimes \widehat{\mathfrak{I}}_{-n-1}+\mathfrak{I}_{n} \otimes \widehat{\mathfrak{C}}_{-n-1}\right),
$$

this means we have to match (3.13) to

$$
\begin{aligned}
r_{12}\left|\phi_{1}^{a} \phi_{2}^{b}\right\rangle & =\sum\left(C_{n} \widehat{I}_{-n-1}+I_{n} \widehat{C}_{-n-1}\right)\left|\phi_{1}^{a} \phi_{2}^{b}\right\rangle+\cdots, \\
r_{12}\left|\psi_{1}^{\alpha} \psi_{2}^{\beta}\right\rangle & =\sum\left(-C_{n} \widehat{I}_{-n-1}-I_{n} \widehat{C}_{-n-1}\right)\left|\psi_{1}^{\alpha} \psi_{2}^{\beta}\right\rangle+\cdots, \\
r_{12}\left|\phi_{1}^{a} \psi_{2}^{\beta}\right\rangle & =\sum\left(-C_{n} \widehat{I}_{-n-1}+I_{n} \widehat{C}_{-n-1}\right)\left|\phi_{1}^{a} \psi_{2}^{\beta}\right\rangle+\cdots, \\
r_{12}\left|\psi_{1}^{\alpha} \phi_{2}^{b}\right\rangle & =\sum\left(C_{n} \widehat{I}_{-n-1}-I_{n} \widehat{C}_{-n-1}\right)\left|\psi_{1}^{\alpha} \phi_{2}^{b}\right\rangle+\cdots,
\end{aligned}
$$


up to an overall shift. Here the first factors $C_{n}$ or $I_{n}$ are understood in the representation labeled by $x_{1}$ while the second factors $\widehat{I}_{-n-1}$ or $\widehat{C}_{-n-1}$ are in the $x_{2}$ one. For this to be possible, we need a rather non-trivial identity:

$$
\begin{gathered}
\frac{\left(x_{1}^{2}+x_{2}^{2}\right)\left(x_{1}^{2} x_{2}^{2}+1\right)-4 x_{1}^{2} x_{2}^{2}}{\left(x_{2}-x_{1}\right)\left(x_{1} x_{2}-1\right)\left(x_{1}^{2}-1\right)\left(x_{2}^{2}-1\right)}+\frac{2 x_{1} x_{2}}{\left(x_{2}-x_{1}\right)\left(x_{1} x_{2}-1\right)} \\
=\frac{x_{2}\left(x_{2}+x_{1}\right)}{\left(x_{2}-x_{1}\right)\left(x_{2}^{2}-1\right)}+\frac{x_{1}\left(x_{2}+x_{1}\right)}{\left(x_{2}-x_{1}\right)\left(x_{1}^{2}-1\right)} .
\end{gathered}
$$

In fact, this identity holds! Subtracting half of the above quantity to normalize the classical r-matrix properly, we find 3

$$
\begin{aligned}
r_{12}\left|\phi_{1}^{a} \phi_{2}^{b}\right\rangle & =\frac{\left(x_{1}^{2}+x_{2}^{2}\right)\left(x_{1}^{2} x_{2}^{2}+1\right)-4 x_{1}^{2} x_{2}^{2}}{2\left(x_{2}-x_{1}\right)\left(x_{1} x_{2}-1\right)\left(x_{1}^{2}-1\right)\left(x_{2}^{2}-1\right)}\left|\phi_{1}^{a} \phi_{2}^{b}\right\rangle+\cdots, \\
r_{12}\left|\psi_{1}^{\alpha} \psi_{2}^{\beta}\right\rangle & =-\frac{\left(x_{1}^{2}+x_{2}^{2}\right)\left(x_{1}^{2} x_{2}^{2}+1\right)-4 x_{1}^{2} x_{2}^{2}}{2\left(x_{2}-x_{1}\right)\left(x_{1} x_{2}-1\right)\left(x_{1}^{2}-1\right)\left(x_{2}^{2}-1\right)}\left|\psi_{1}^{\alpha} \psi_{2}^{\beta}\right\rangle+\cdots, \\
r_{12}\left|\phi_{1}^{a} \psi_{2}^{\beta}\right\rangle & =-\frac{\left(x_{1} x_{2}+1\right)\left(x_{2}+x_{1}\right)}{2\left(x_{1}^{2}-1\right)\left(x_{2}^{2}-1\right)}\left|\phi_{1}^{a} \psi_{2}^{\beta}\right\rangle+\cdots, \\
r_{12}\left|\psi_{1}^{\alpha} \phi_{2}^{b}\right\rangle & =\frac{\left(x_{1} x_{2}+1\right)\left(x_{2}+x_{1}\right)}{2\left(x_{1}^{2}-1\right)\left(x_{2}^{2}-1\right)}\left|\psi_{1}^{\alpha} \phi_{2}^{b}\right\rangle+\cdots,
\end{aligned}
$$

with

$$
\begin{aligned}
\sum_{n=0}^{\infty} C_{n} \widehat{I}_{-n-1} & =\frac{x_{1}^{2}\left(x_{2}^{2}-1\right)}{2\left(x_{2}-x_{1}\right)\left(x_{1} x_{2}-1\right)\left(x_{1}^{2}-1\right)}, \\
\sum_{n=0}^{\infty} I_{n} \widehat{C}_{-n-1} & =\frac{x_{2}^{2}\left(x_{1}^{2}-1\right)}{2\left(x_{2}-x_{1}\right)\left(x_{1} x_{2}-1\right)\left(x_{2}^{2}-1\right)} .
\end{aligned}
$$

Choosing

$$
\begin{aligned}
& C_{n}=\widehat{C}_{n}=\frac{x^{n+1}+x^{-n-1}}{2\left(x-x^{-1}\right)}, \\
& I_{n}=\widehat{I}_{n}=\frac{1}{2}\left(x^{n}-x^{-n}\right),
\end{aligned}
$$

we find that formula (3.17) holds. Again, there are ambiguities in rescaling $C_{n}$ and $I_{n}$. Our definition is motivated by the commutation relations in the next section. Note that $I_{0}$ vanishes identically, which is expected from the argument in the introduction.

\section{Commutation relations}

In the previous section we have rewritten the classical r-matrix in terms of generators of a tentative Yangian double. In the process, we have defined level-n operators $\mathfrak{Q}_{a, n}^{\alpha}, \mathfrak{S}^{a}{ }_{\alpha, n}$,

\footnotetext{
${ }^{3}$ It would be interesting to understand the relation between this subtraction and the dressing factor.
} 
$\mathfrak{R}_{b, n}^{a}, \mathfrak{L}_{\beta, n}^{\alpha}, \mathfrak{C}_{n}$ (and $\mathfrak{I}_{n}$ ) and their duals, which reduce to the original $\mathfrak{s u}(2 \mid 2)$ generators $\mathfrak{Q}^{\alpha}{ }_{a}$, $\mathfrak{S}^{a}{ }_{\alpha}, \mathfrak{R}^{a}{ }_{b}, \mathfrak{L}^{\alpha}{ }_{\beta}, \mathfrak{C}$ at level-zero. Here we would like to investigate their commutation relations.

Originally the operators $\mathfrak{Q}^{\alpha}{ }_{a, n}, \mathfrak{S}^{a}{ }_{\alpha, n}, \mathfrak{C}_{n}$ and $\mathfrak{I}_{n}$ are defined only for $n \geq 0$ while their duals are defined only for $n<0$. Since both these operators and their duals share the same expressions as can be seen in (3.4), (3.5), (3.22) and (3.23), let us combine the formula by extending their definition for $n<0$. On the other hand, the duals of the operators $\mathfrak{R}_{b, n}^{a}$ (3.11) and $\mathfrak{L}_{\beta, n}^{\alpha}(3.12)$ can be obtained from the original operators by substituting $n$ with $-n$. We will not consider them in the commutation relations. To summarize, we would like to study the commutation relations between the operators $\mathfrak{Q}_{a, n}^{\alpha}, \mathfrak{S}^{a}{ }_{\alpha, n}, \mathfrak{R}_{b, n}^{a}, \mathfrak{L}_{\beta, n}^{\alpha}, \mathfrak{C}_{n}$ and $\mathfrak{I}_{n}$, where the indices run over positive and negative integers.

We would like to remark that at the present stage it is difficult to exclude that the following commutation relations could be accidental to our representation, and need to be modified later. In particular, it is impossible from (3.11), (3.12), (3.22) and (3.23) to distinguish between $\mathfrak{R}_{b, n}^{a}$ and $-\mathfrak{R}_{b,-n-2}^{a}$, between $\mathfrak{L}^{\alpha}{ }_{\beta, n}$ and $-\mathfrak{L}_{\beta,-n-2}^{\alpha}$, between $\mathfrak{C}_{n}$ and $\mathfrak{C}_{-n-2}$ and between $\mathfrak{I}_{n}$ and $-\mathfrak{I}_{-n}$. Here we have chosen to present them in the most compact form as we could find.

Acting the operators $\left\{\mathfrak{Q}_{a, m}^{\alpha}, \mathfrak{S}_{\beta, n}^{b}\right\}$ on the bosonic state $\left|\phi^{c}\right\rangle$ and the fermionic state $\left|\psi^{\gamma}\right\rangle$ respectively using (2.11) and (2.12) and reinterpreting the result as the action of a single bosonic operator with the help of (2.9) and (2.10), we find

$$
\left\{\mathfrak{Q}_{a, m}^{\alpha}, \mathfrak{S}_{\beta, n}^{b}\right\}=\delta_{a}^{b} \mathfrak{L}_{\beta, m+n}^{\alpha}+\delta_{\beta}^{\alpha} \mathfrak{R}_{a, m+n}^{b}+\delta_{a}^{b} \delta_{\beta}^{\alpha} \mathfrak{C}_{m+n} .
$$

This is the higher level analogue of the commutation relation:

$$
\left\{\mathfrak{Q}^{\alpha}{ }_{a}, \mathfrak{S}_{\beta}^{b}\right\}=\delta_{a}^{b} \mathfrak{L}_{\beta}^{\alpha}+\delta_{\beta}^{\alpha} \mathfrak{R}_{a}^{b}+\delta_{a}^{b} \delta_{\beta}^{\alpha} \mathfrak{C} .
$$

This result justifies our definition of $\mathfrak{R}_{b, n}^{a}, \mathfrak{L}^{\alpha}{ }_{\beta, n}$ and $\mathfrak{C}_{n}$ in (3.11), (3.12) and (3.22). Similarly, we find

$$
\begin{aligned}
& \left\{\mathfrak{Q}^{\alpha}{ }_{a, m}, \mathfrak{Q}_{b, n}^{\beta}\right\}=\frac{2 \zeta \alpha}{i}\left[\epsilon^{\alpha \beta} \epsilon_{a b} \mathfrak{C}_{m-n-1}+\epsilon^{\alpha \beta} \epsilon_{c\{a} \mathfrak{R}_{b\}, m-n-1}^{c}+\epsilon_{a b} \epsilon^{\gamma\{\alpha} \mathfrak{L}^{\beta\}}{ }_{\gamma, m-n-1}\right], \\
& \left\{\mathfrak{S}_{\alpha, m}^{a}, \mathfrak{S}_{\beta, n}^{b}\right\}=\frac{i}{2 \zeta \alpha}\left[\epsilon^{a b} \epsilon_{\alpha \beta} \mathfrak{C}_{m-n-1}+\epsilon_{\alpha \beta} \epsilon^{c\{a} \mathfrak{R}_{c, m-n-1}^{b\}}+\epsilon^{a b} \epsilon_{\gamma\{\alpha} \mathfrak{L}_{\beta\}, m-n-1}^{\gamma}\right],
\end{aligned}
$$

where parentheses enclosing indices denote symmetrization (dividing by two). In the computation the following formula is useful.

$$
\epsilon^{a b} \epsilon_{c d}=\delta_{c}^{a} \delta_{d}^{b}-\delta_{d}^{a} \delta_{c}^{b}, \quad \delta_{a}^{d} \epsilon_{b c}+\delta_{b}^{d} \epsilon_{c a}+\delta_{c}^{d} \epsilon_{a b}=0
$$

At level zero, one recovers from (4.3), (4.4), (3.22) and (2.14) the two central extensions $\mathfrak{P}=a b$ and $\mathfrak{K}=c d$ of the superalgebra $\mathfrak{s u}(2 \mid 2)$. The reader might find unpleasant the appearance of the factor $2 \zeta \alpha$ in the commutation relations. We can always get rid of it by rescaling the generators by $\mathfrak{Q}_{a, n}^{\alpha} \rightarrow \mathfrak{Q}^{\alpha}{ }_{a, n} / \sqrt{2 \zeta \alpha}$ and $\mathfrak{S}^{a}{ }_{\alpha, n} \rightarrow \mathfrak{S}^{a}{ }_{\alpha, n} \sqrt{2 \zeta \alpha}$. 
The commutation relations between one bosonic and one fermionic operator read

$$
\begin{aligned}
& {\left[\mathfrak{R}_{b, m}^{a}, \mathfrak{Q}^{\gamma}{ }_{c, n}\right]=\operatorname{sign}(m+1) \sum_{l=-|m+1|+1}^{|m+1|-1}\left(-\delta_{c}^{a} \mathfrak{Q}_{b, l+n}^{\gamma}+\frac{1}{2} \delta_{b}^{a} \mathfrak{Q}_{c, l+n}^{\gamma}\right),} \\
& {\left[\mathfrak{R}_{b, m}^{a}, \mathfrak{S}_{\gamma, n}^{c}\right]=\operatorname{sign}(m+1) \sum_{l=-|m+1|+1}^{|m+1|-1}\left(\delta_{b}^{c} \mathfrak{S}^{a}{ }_{\gamma, l+n}-\frac{1}{2} \delta_{b}^{a} \mathfrak{S}_{\gamma, l+n}^{c}\right),} \\
& {\left[\mathfrak{L}_{\beta, m}^{\alpha}, \mathfrak{Q}^{\gamma}{ }_{c, n}\right]=\operatorname{sign}(m+1) \sum_{l=-|m+1|+1}^{|m+1|-1}\left(\delta_{\beta}^{\gamma} \mathfrak{Q}^{\alpha}{ }_{c, l+n}-\frac{1}{2} \delta_{\beta}^{\alpha} \mathfrak{Q}_{c, l+n}^{\gamma}\right),} \\
& {\left[\mathfrak{L}_{\beta, m}^{\alpha}, \mathfrak{S}_{\gamma, n}^{c}\right]=\operatorname{sign}(m+1) \sum_{l=-|m+1|+1}^{|m+1|-1},\left(-\delta_{\gamma}^{\alpha} \mathfrak{S}_{\beta, l+n}^{c}+\frac{1}{2} \delta_{\beta}^{\alpha} \mathfrak{S}_{\gamma, l+n}^{c}\right) .}
\end{aligned}
$$

Here we have to expand the $q$-number $[m+1]_{x}$ attached to the bosonic operators $\mathfrak{R}_{b, m}^{a}$ and $\mathfrak{L}^{\alpha}{ }_{\beta, m}$ by

$$
[m]_{x}=\operatorname{sign}(m) \sum_{l=-|m|+1}^{|m|-1}, x^{l},
$$

because only monomials are attached to the fermionic operators $\mathfrak{Q}^{\gamma}{ }_{c, n}$ and $\mathfrak{S}^{c}{ }_{\gamma, n}$. Note that $\operatorname{sign}(n)$ is defined to be $1,0,-1$ for $n>0, n=0, n<0$ respectively, and the prime ' in the summation symbol $\sum^{\prime}$ indicates that the summation is taken by steps of two.

Now let us turn to the commutation relations between two bosonic operators.

$$
\begin{aligned}
& {\left[\mathfrak{R}_{b, m}^{a}, \mathfrak{R}_{d, n}^{c}\right]=\operatorname{sign}(m+1)(n+1) \sum_{l=|| m+1|-| n+1||}^{|m+1|+|n+1|-2}\left(\delta_{b}^{c} \mathfrak{R}_{d, l}^{a}-\delta_{d}^{a} \mathfrak{R}_{b, l}^{c}\right),} \\
& {\left[\mathfrak{L}_{\beta, m}^{\alpha}, \mathfrak{L}^{\gamma}{ }_{\delta, n}\right]=\operatorname{sign}(m+1)(n+1) \sum_{l=|| m+1|-| n+1||}^{|m+1|+|n+1|-2}\left(\delta_{\beta}^{\gamma} \mathfrak{L}_{\delta, l}^{\alpha}-\delta_{\delta}^{\alpha} \mathfrak{L}_{\beta, l}^{\gamma}\right) .}
\end{aligned}
$$

Here we have to expand the product of two $q$-numbers in terms of the following summation of q-numbers:

$$
[m]_{x}[n]_{x}=\operatorname{sign}(m n) \sum_{l=|| m|-| n||+1}^{|m|+|n|-1}[l]_{x} .
$$

Finally the commutation relations between the fermionic operators $\mathfrak{Q}_{a, m}^{\alpha}$ and $\mathfrak{S}^{a}{ }_{\alpha, m}$ and our parity operator $\mathfrak{I}_{n}$ are non-trivial:

$$
\begin{aligned}
& {\left[\mathfrak{Q}^{\alpha}{ }_{a, m}, \mathfrak{I}_{n}\right]=\mathfrak{Q}^{\alpha}{ }_{a, m+n}-\mathfrak{Q}^{\alpha}{ }_{a, m-n},} \\
& {\left[\mathfrak{S}^{a}{ }_{\alpha, m}, \mathfrak{I}_{n}\right]=-\mathfrak{S}^{a}{ }_{\alpha, m+n}+\mathfrak{S}^{a}{ }_{\alpha, m-n} .}
\end{aligned}
$$




\section{Conclusions}

We have expressed the classical r-matrix of the AdS/CFT correspondence in terms of a Yangian double, or an infinite series of tensor products of operators. We have also studied the commutation relations among these new generators. We hope our result will clarify the underlying symmetry, and give a clue to the construction of the universal R-matrix of the model.

We shall list some of the main future directions to prosecute our work.

- The most important development will be to obtain along these lines a universal expression for the full quantum R-matrix. We believe that the formula in this paper can be rather suggestive of the appropriate completion, but a full derivation is still to be worked out.

- The appropriate coproduct and Hopf algebra structure have to be defined for the generators we constructed, in order to study the infinite dimensional symmetry of the R-matrix. This is traditionally presented in the Chevalley basis, rather than in the Cartan-Weyl one.

- One should make contact with Beisert's formulation of the Yangian symmetry given in Drinfeld's first realization in [13], and show the relationship with the one presented in this paper.

- The question whether our expression (1.3) is truly "universal" can also be addressed by studying the double structure of the classical r-matrix for the bound states [14]. We would like to see whether the classical r-matrix for the bound states can also be rewritten as the same Yangian double satisfying the same algebra.

- So far the main results on the integrable structure of the dilatation operator in the Super Yang-Mills theory are restricted to the sector of the single trace operators or the single string states. It would be interesting if this integrable structure can be lifted to multi-trace operators or multi-string states. The correspondence between the symmetry generators of matrix string theory (gauge theory) and those of light-cone string field theory on the flat space (string theory) given recently in 25] may give a clue to this question.

\section{Acknowledgments}

We thank P. Etingof for enlightening discussions. We would also like to thank T. Matsumoto and F. Spill for many interesting discussions and helpful email exchange. This work is supported in part by funds provided by the U.S. Department of Energy (D.O.E.) under cooperative research agreement DE-FG02-05ER41360. The work of S.M. is supported partly by 
Nishina Memorial Foundation, Inamori Foundation and Grant-in-Aid for Young Scientists (\#18740143) from the Japan Ministry of Education, Culture, Sports, Science and Technology. A.T. thanks Istituto Nazionale di Fisica Nucleare (I.N.F.N.) for supporting him through a "Bruno Rossi" postdoctoral fellowship.

\section{References}

[1] J. A. Minahan and K. Zarembo, "The Bethe-ansatz for $\mathrm{N}=4$ super Yang-Mills," JHEP 0303 (2003) 013 arXiv:hep-th/0212208]. • N. Beisert, C. Kristjansen and M. Staudacher, "The dilatation operator of N = 4 super Yang-Mills theory," Nucl. Phys. B 664 (2003) 131 arXiv:hep-th/0303060|. - N. Beisert, "The complete one-loop dilatation operator of $\mathrm{N}=$ 4 super Yang-Mills theory," Nucl. Phys. B 676 (2004) 3 arXiv:hep-th/0307015]. • N. Beisert and M. Staudacher, "The N = 4 SYM integrable super spin chain," Nucl. Phys. B 670 (2003) 439 arXiv:hep-th/0307042]. • L. Dolan, C. R. Nappi and E. Witten, "A relation between approaches to integrability in superconformal Yang-Mills theory," JHEP 0310 (2003) 017 [arXiv:hep-th/0308089]. • N. Beisert, "The su(2|3) dynamic spin chain," Nucl. Phys. B 682 (2004) 487 [arXiv:hep-th/0310252]. • L. Dolan, C. R. Nappi and E. Witten, "Yangian symmetry in D = 4 superconformal Yang-Mills theory," arXiv:hep-th/0401243. - N. Beisert, V. Dippel and M. Staudacher, "A novel long range spin chain and planar N = 4 super Yang-Mills," JHEP 0407 (2004) 075 arXiv:hep-th/0405001]. • M. Staudacher, "The factorized S-matrix of CFT/AdS," JHEP 0505 (2005) 054 arXiv:hep-th/0412188. - N. Beisert and M. Staudacher, "Long-range PSU(2,2|4) Bethe ansaetze for gauge theory and strings," Nucl. Phys. B 727 (2005) 1 [arXiv:hep-th/0504190].

[2] I. Bena, J. Polchinski and R. Roiban, "Hidden symmetries of the $\operatorname{AdS}(5) \times \mathrm{S}^{* *} 5$ superstring," Phys. Rev. D 69 (2004) 046002 arXiv:hep-th/0305116]. • G. Arutyunov, S. Frolov, J. Russo and A. A. Tseytlin, "Spinning strings in $\operatorname{AdS}(5) \times \mathrm{S}^{* *} 5$ and integrable systems," Nucl. Phys. B 671 (2003) 3 arXiv:hep-th/0307191]. • G. Arutyunov, J. Russo and A. A. Tseytlin, "Spinning strings in $\operatorname{AdS}(5) \times \mathrm{S}^{* *} 5$ : New integrable system relations," Phys. Rev. D 69 (2004) 086009 arXiv:hep-th/0311004]. • V. A. Kazakov, A. Marshakov, J. A. Minahan and K. Zarembo, "Classical / quantum integrability in AdS/CFT," JHEP 0405 (2004) 024 [arXiv:hep-th/0402207]. • G. Arutyunov and S. Frolov, "Integrable Hamiltonian for classical strings on AdS(5) x S**5," JHEP 0502 (2005) 059 arXiv:hep-th/0411089 - • L. F. Alday, G. Arutyunov and A. A. Tseytlin, "On integrability of classical superstrings in $\operatorname{AdS}(5) \times \mathrm{S}^{* *}$," JHEP 0507 (2005) 002 arXiv:hep-th/0502240]. • S. Frolov, J. Plefka and M. Zamaklar, "The AdS(5) x S**5 superstring in light-cone gauge and its Bethe equations," J. Phys. A 39 (2006) 13037

arXiv:hep-th/0603008 . • T. Klose and K. Zarembo, "Bethe ansatz in stringy sigma models," J. Stat. Mech. 0605 (2006) P006 arXiv:hep-th/0603039|. • G. Arutyunov, 
S. Frolov, J. Plefka and M. Zamaklar, "The off-shell symmetry algebra of the lightcone AdS(5) x S**5 superstring," J. Phys. A 40 (2007) 3583 arXiv:hep-th/0609157. arXiv:hep-th/0609157. • T. Klose, T. McLoughlin, R. Roiban and K. Zarembo, "Worldsheet scattering in AdS(5) x S**5," JHEP 0703 (2007) 094 arXiv:hep-th/0611169. D. M. Hofman and J. M. Maldacena, "Giant magnons," J. Phys. A 39 (2006) 13095 arXiv:hep-th/0604135. • M. Spradlin and A. Volovich, "Dressing the giant magnon," JHEP 0610, 012 (2006) arXiv:hep-th/0607009|. • J. Maldacena and I. Swanson, "Connecting giant magnons to the pp-wave: An interpolating limit of $\operatorname{AdS}(5) \times \mathrm{S}^{* *}$," arXiv:hep-th/0612079. • J. A. Minahan, "Zero modes for the giant magnon," JHEP 0702 (2007) 048 arXiv:hep-th/0701005]. • G. Papathanasiou and M. Spradlin, "Semiclassical Quantization of the Giant Magnon," arXiv:0704.2389 [hep-th]. • M. de Leeuw, "Coordinate Bethe Ansatz for the String S-Matrix," arXiv:0705.2369 [hep-th].

[3] N. Beisert, "The dilatation operator of $\mathrm{N}=4$ super Yang-Mills theory and integrability," Phys. Rept. 405 (2005) 1 arXiv:hep-th/0407277. • J. Plefka, "Spinning strings and integrable spin chains in the AdS/CFT correspondence," arXiv:hep-th/0507136. J. A. Minahan, "A Brief Introduction To The Bethe Ansatz In N=4 Super-Yang-Mills," J. Phys. A 39, 12657 (2006).

[4] N. Beisert, "The su(2|2) dynamic S-matrix," arXiv:hep-th/0511082.

[5] N. Beisert, "The Analytic Bethe Ansatz for a Chain with Centrally Extended su(2|2) Symmetry," J. Stat. Mech. 0701, P017 (2007) arXiv:nlin.si/0610017].

[6] G. Arutyunov, S. Frolov and M. Staudacher, "Bethe ansatz for quantum strings," JHEP 0410 (2004) 016 arXiv:hep-th/0406256. • S. Schäfer-Nameki, M. Zamaklar and K. Zarembo, "Quantum corrections to spinning strings in $\operatorname{AdS}(5) \times \mathrm{S}^{* * 5}$ and Bethe ansatz: A comparative study," JHEP 0509 (2005) 051 arXiv:hep-th/0507189. - S. Schäfer-Nameki and M. Zamaklar, "Stringy sums and corrections to the quantum string Bethe ansatz," JHEP 0510 (2005) 044 arXiv:hep-th/0509096]. • N. Beisert and A. A. Tseytlin, "On quantum corrections to spinning strings and Bethe equations," Phys. Lett. B 629 (2005) 102 arXiv:hep-th/0509084]. • N. Beisert and T. Klose, "Long-range gl(n) integrable spin chains and plane-wave matrix theory," J. Stat. Mech. 0607 (2006) P006 arXiv:hep-th/0510124. • R. Hernandez and E. Lopez, "Quantum corrections to the string Bethe ansatz," JHEP 0607 (2006) 004 [arXiv:hep-th/0603204]. • L. Freyhult and C. Kristjansen, "A universality test of the quantum string Bethe ansatz," Phys. Lett. B 638 (2006) 258 arXiv:hep-th/0604069.

[7] R. A. Janik, "The AdS(5) x $\mathrm{S}^{* *} 5$ superstring worldsheet S-matrix and crossing symmetry," Phys. Rev. D 73 (2006) 086006 [arXiv:hep-th/0603038].

[8] G. Arutyunov and S. Frolov, "On $\operatorname{AdS}(5)$ x S**5 string S-matrix," Phys. Lett. B 639 (2006) 378 arXiv:hep-th/0604043. 
[9] N. Beisert, "On the scattering phase for AdS(5) x S**5 strings," Mod. Phys. Lett. A 22, 415 (2007) arXiv:hep-th/0606214. - N. Beisert, R. Hernandez and E. Lopez, "A crossing-symmetric phase for $\operatorname{AdS}(5)$ x S**5 strings," JHEP 0611 (2006) 070 arXiv:hep-th/0609044. • Z. Bern, M. Czakon, L. J. Dixon, D. A. Kosower and V. A. Smirnov, "The Four-Loop Planar Amplitude and Cusp Anomalous Dimension in Maximally Supersymmetric Yang-Mills Theory," Phys. Rev. D 75, 085010 (2007) arXiv:hep-th/0610248. • N. Beisert, B. Eden and M. Staudacher, "Transcendentality and crossing," J. Stat. Mech. 0701, P021 (2007) arXiv:hep-th/0610251]. • C. Gomez and R. Hernandez, "Integrability and non-perturbative effects in the AdS/CFT correspondence," Phys. Lett. B 644 (2007) 375 arXiv:hep-th/0611014. • M. K. Benna, S. Benvenuti, I. R. Klebanov and A. Scardicchio, "A test of the AdS/CFT correspondence using high-spin operators," Phys. Rev. Lett. 98, 131603 (2007) arXiv:hep-th/0611135. - A. V. Kotikov and L. N. Lipatov, "On the highest transcendentality in N = 4 SUSY," Nucl. Phys. B 769, 217 (2007) arXiv:hep-th/0611204. • A. Rej, M. Staudacher and S. Zieme, "Nesting and dressing," arXiv:hep-th/0702151. • K. Sakai and Y. Satoh, "Origin of dressing phase in N=4 Super Yang-Mills," arXiv:hep-th/0703177. • N. Gromov and P. Vieira, "Constructing the AdS/CFT dressing factor," arXiv:hep-th/0703266. A. V. Kotikov, L. N. Lipatov, A. Rej, M. Staudacher and V. N. Velizhanin, "Dressing and Wrapping," arXiv:0704.3586 [hep-th].

[10] A. Agarwal, "Comments on higher loop integrability in the $\operatorname{su}(1 \mid 1)$ sector of $\mathrm{N}=4$ SYM: Lessons from the su(2) sector," arXiv:hep-th/0506095. • B. I. Zwiebel, "Yangian symmetry at two-loops for the su(2|1) sector of $\mathrm{N}=4$ SYM," J. Phys. A 40, 1141 (2007) arXiv:hep-th/0610283.

[11] G. Arutyunov, S. Frolov and M. Zamaklar, "The Zamolodchikov-Faddeev algebra for AdS(5) x S**5 superstring," JHEP 0704, 002 (2007) arXiv:hep-th/0612229.

[12] M. Hatsuda and K. Yoshida, "Classical integrability and super Yangian of superstring on AdS(5) x S**5," Adv. Theor. Math. Phys. 9, 703 (2005) arXiv:hep-th/0407044. • A. Das, J. Maharana, A. Melikyan and M. Sato, "The algebra of transition matrices for the AdS(5) x S**5 superstring," JHEP 0412, 055 (2004) [arXiv:hep-th/0411200]. • A. Das, A. Melikyan and M. Sato, "The algebra of flat currents for the string on AdS(5) x S**5 in the light-cone gauge," JHEP 0511, 015 (2005) arXiv:hep-th/0508183. • C. Gomez and R. Hernandez, "Quantum deformed magnon kinematics," JHEP 0703, 108 (2007) arXiv:hep-th/0701200. • M. J. Martins and C. S. Melo, "The Bethe ansatz approach for factorizable centrally extended S-matrices," arXiv:hep-th/0703086. • B. . J. Stefanski, "Landau-Lifshitz sigma-models, fermions and the AdS/CFT correspondence," arXiv:0704.1460 [hep-th]. • C. A. S. Young, "q-deformed supersymmetry and dynamic magnon representations," arXiv:0704.2069 [hep-th]. 
[13] N. Beisert, "The S-Matrix of AdS/CFT and Yangian Symmetry," PoS SOLVAY, 002 (2006) arXiv:0704.0400 [nlin.SI].

[14] N. Dorey, "Magnon bound states and the AdS/CFT correspondence," J. Phys. A 39 (2006) 13119 arXiv:hep-th/0604175. • H. Y. Chen, N. Dorey and K. Okamura, "Dyonic giant magnons," JHEP 0609 (2006) 024 |arXiv:hep-th/0605155]. • H. Y. Chen, N. Dorey and K. Okamura, "On the scattering of magnon boundstates," JHEP 0611 (2006) 035 arXiv:hep-th/0608047|. • R. Roiban, "Magnon bound-state scattering in gauge and string theory," JHEP 0704, 048 (2007) arXiv:hep-th/0608049. • H. Y. Chen, N. Dorey and K. Okamura, "The asymptotic spectrum of the $\mathrm{N}=4$ super Yang-Mills spin chain," JHEP 0703, 005 (2007) arXiv:hep-th/0610295]. • N. Dorey, D. M. Hofman and J. Maldacena, "On the singularities of the magnon S-matrix," arXiv:hep-th/0703104.

[15] C. Gomez and R. Hernandez, "The magnon kinematics of the AdS/CFT correspondence," JHEP 0611 (2006) 021 arXiv:hep-th/0608029|. • J. Plefka, F. Spill and A. Torrielli, "On the Hopf algebra structure of the AdS/CFT S-matrix," Phys. Rev. D 74 (2006) 066008 arXiv:hep-th/0608038.

[16] A. A. Belavin and V. G. Drinfeld, "Solutions of the classical Yang-Baxter equation for simple Lie algebras," Funct. Anal. Appl. 16 (1982), 159. • A. A. Belavin and V. G. Drinfeld, "Classical Yang-Baxter equation for simple Lie algebras," Funct. Anal. Appl. 17 (1983) 220. • A. A. Belavin and V. G. Drinfeld, "Triangle equation for simple Lie algebras," Mathematical Physics Reviews (ed. Novikov at al.) Harwood, New York (1984), 93. • V. G. Drinfeld, "Quantum groups," Proc. of the International Congress of Mathematicians, Berkeley, 1986, American Mathematical Society, 1987, 798. • D. A Leites and V. V. Serganova, "Solutions of the classical Yang-Baxter equation for simple superalgebras," Theor. Math. Phys. 58 (1984) 16. • R. B. Zhang, M. D. Gould and A. J. Bracken, "Solutions of the graded classical Yang-Baxter equation and integrable models," J. Phys. A: Math. Gen. 24 (1991) 1185. • G. Karaali, "Constructing r-matrices on simple Lie superalgebras," J. Algebra 282 (2004) 83 [arXiv:math.QA/0303246]. G. Karaali, "A New Lie Bialgebra Structure on sl(2,1)," Contemp. Math. 413 (2006) 101 arXiv:math.RA/0410473.

[17] P. Etingof and O. Schiffman, "Lectures on Quantum Groups," Lectures in Mathematical Physics, International Press, Boston, 1998. • V. Chari and A. Pressley, "A Guide To Quantum Groups," Cambridge, UK: Univ. Press (1994).

[18] A. Torrielli, "Classical r-matrix of the su(2|2) SYM spin-chain," Phys. Rev. D 75 (2007) 105020 [arXiv:hep-th/0701281].

[19] V. G. Drinfeld, "A new realization of Yangians and quantized affine algebras," Sov. Math. Dokl. 36 (1988) 212. 
[20] S. M. Khoroshkin and V. N. Tolstoy, "Universal R-matrix for quantized (super)algebras," Commun. Math. Phys. 141 (1991) 599. • H. Yamane, "Universal R-matrices for quantum groups associated to simple Lie superalgebras," Proc. Japan Acad. Ser. A 67 (1991) 108. • H. Yamane, "Quantized enveloping algebras associated with simple Lie superalgebras and their universal R-matrices," Publ. Res. Math. Inst. Sci. 30 (1994) 15. • S. M. Khoroshkin and V. N. Tolstoy, "Yangian Double And Rational R Matrix," arXiv:hep-th/9406194. - H. Yamane, "On defining relations of affine Lie superalgebras and affine quantized universal enveloping superalgebras," Publ. Res. Math. Inst. Sci. 35 (1999) 321, 37 (2001) 615 (Errata). |arXiv:q-alg/9603015|. • V. Stukopin, "Quantum Double of Yangian of Lie Superalgebra A(m,n) and computation of Universal R-matrix," arXiv:math/0504302.

[21] I. Heckenberger, F. Spill, A. Torrielli and H. Yamane, "Drinfeld second realization of the quantum affine superalgebras of $D^{(1)}(2,1 ; x)$ via the Weyl groupoid," arXiv:0705.1071 [math.QA].

[22] F. Spill, Diploma Thesis, Humboldt University of Berlin, 2007.

[23] R. M. Gade, "Universal R-matrix and graded Hopf algebra structure of $U_{q}(\widehat{g l}(2 \mid 2))$," J. Phys. A 31 (1998) 4909.

[24] D. Berenstein, J. M. Maldacena and H. Nastase, "Strings in flat space and pp waves from N = 4 super Yang Mills," JHEP 0204 (2002) 013 [arXiv:hep-th/0202021]. • C. G. Callan, H. K. Lee, T. McLoughlin, J. H. Schwarz, I. J. Swanson and X. Wu, "Quantizing string theory in AdS(5) x S**5: Beyond the pp-wave," Nucl. Phys. B 673 (2003) 3 arXiv:hep-th/0307032| • C. G. Callan, T. McLoughlin and I. J. Swanson, "Holography beyond the Penrose limit," Nucl. Phys. B 694 (2004) 115 arXiv:hep-th/0404007. • C. G. Callan, T. McLoughlin and I. J. Swanson, "Higher impurity AdS/CFT correspondence in the near-BMN limit," Nucl. Phys. B 700 (2004) 271 arXiv:hep-th/0405153. • A. Hentschel, J. Plefka and P. Sundin, "Testing the nested light-cone Bethe equations of the $\operatorname{AdS}(5)$ x $\mathrm{S}^{* *} 5$ superstring," arXiv:hep-th/0703187.

[25] R. Dijkgraaf and L. Motl, "Matrix string theory, contact terms, and superstring field theory," arXiv:hep-th/0309238. • S. Moriyama, "Comments on supersymmetry algebra and contact term in matrix string theory," JHEP 0409, 013 (2004) arXiv:hep-th/0405091. - I. Kishimoto, S. Moriyama and S. Teraguchi, "Twist field as three string interaction vertex in light cone string field theory," Nucl. Phys. B 744, 221 (2006) arXiv:hep-th/0603068. • I. Kishimoto and S. Moriyama, "On LCSFT/MST correspondence," arXiv:hep-th/0611113. 